\title{
An obstacle-problem-like equation with two phases: pointwise regularity of the solution and an estimate of the Hausdorff dimension of the free boundary
}

\author{
G. S. WEISS ${ }^{\dagger}$ \\ Graduate School of Mathematical Sciences, University of Tokyo, 3-8-1 Komaba, Meguro-ku, \\ Tokyo-to, 153-8914 Japan
}

[Received 22 April 1999 and in revised form 2 October 2000]

\begin{abstract}
Concerning the obstacle-problem-like equation $\Delta u=\frac{\lambda_{+}}{2} \chi_{\{u>0\}}-\frac{\lambda_{-}}{2} \chi_{\{u<0\}}$, where $\lambda_{+} \geqslant 0$ and $\lambda_{+}+\lambda_{-}>0$, we prove regularity of the solution as well as an estimate on the Hausdorff dimension of the free boundary $\Omega \cap(\partial\{u>0\} \cup \partial\{u<0\})$. We apply this result to the Stefan-like equation $\alpha \partial_{t} \max (v, 0)+\beta \partial_{t} \min (v, 0)-\Delta v=0$.
\end{abstract}

Keywords: Obstacle problem; two-phase problem; free boundary; monotonicity formula; frequency estimate; Hausdorff dimension

\section{Introduction}

Whereas the regularity in one-phase free boundary problems has by now been extensively studied, one-phase methods prove in many cases to be unsuitable for the corresponding two-phase problems. Concerning two-phase problems, even regularity of the solution is in many cases still unknown.

Here we study the regularity of the obstacle-problem-like equation

$$
\Delta u=\frac{\lambda_{+}}{2} \chi_{\{u>0\}}-\frac{\lambda_{-}}{2} \chi_{\{u<0\}},
$$

where $\lambda_{+} \geqslant 0$ and $\lambda_{+}+\lambda_{-}>0$.

Equation (1) is related to the time-dependent equation $0=\alpha \partial_{t} \max (v, 0)+\beta \partial_{t} \min (v, 0)-\Delta v$ in $(0, T) \times \Omega$ which has been used to describe an instantaneous and complete reaction of two substances coming into contact at a surface $\Gamma$ (see [2,3] and [6]). The difficulty one confronts in this Stefan-like problem is that the interface $\{v=0\}$ consists in general of two parts-one where the gradient of $v$ is nonzero and one where the gradient of $v$ vanishes. At the latter part we expect the gradient of $v$ to have linear growth in space. However, because of the decomposition into two different types of growth, it is not possible to derive a growth estimate by, for example, a Bernstein technique.

Assuming that $\alpha>\beta>0$ and that the time derivative $\partial_{t} v$ is non-negative and Hölder continuous near some free boundary point $\left(t_{0}, x_{0}\right), v\left(t_{0}\right)$ is a solution of (1) with Hölder continuous coefficients $\lambda_{+}$and $\lambda_{-}$. To that our result applies and yields the expected $C^{1,1}$-regularity of $v\left(t_{0}\right)$ in a pointwise sense and, for positive time derivative, the Hausdorff dimension estimate $\operatorname{dim}\left(\partial\left\{v\left(t_{0}\right)>0\right\} \cup \partial\left\{v\left(t_{0}\right)<0\right\}\right) \leqslant n-1$ (see Proposition 4.1, Remark 4.1, Corollary 5.1 and Remark 5.1).

\footnotetext{
Email: gw@ms.u-tokyo.ac.jp
} 
Our methods are completely local and rely on new ideas involving frequency estimates as well as a monotonicity formula introduced by the author in [9] and used in [7].

\section{Notation}

Throughout this paper $\mathbb{R}^{n}$ will be equipped with the Euclidean inner product $x \cdot y$ and the induced norm $|x|$, and $B_{r}\left(x_{0}\right)$ will denote the open $n$-dimensional ball of centre $x_{0}$, radius $r$ and volume $r^{n} \omega_{n}$. We will use the $k$-dimensional Hausdorff measure $\mathcal{H}^{k}$ approximated by $\mathcal{H}^{k, \delta}$ which we define as the $H_{k}^{\delta}$ of [5].

When considering a set $A, \chi_{A}$ shall stand for the characteristic function of $A$, while $v$ shall typically denote the outward normal to a given boundary.

\section{Existence}

Let $\lambda_{+} \geqslant 0, \lambda_{+}+\lambda_{-}>0, n \geqslant 2$, let $\Omega$ be a bounded open subset of $\mathbb{R}^{n}$ with Lipschitz boundary, assume that $u_{D} \in H^{1,2}(\Omega)$ and let $A:=\left\{v \in H^{1,2}(\Omega): v-u_{D} \in H_{0}^{1,2}(\Omega)\right\}$. Then the functional $E(v):=\int_{\Omega}\left(|\nabla v|^{2}+\lambda_{+} \max (v, 0)-\lambda_{-} \min (v, 0)\right)$, being real-valued, coercive and weakly lower semi-continuous, attains its infimum on the affine subspace $A$ of $H^{1,2}(\Omega)$ at the point $u \in A$.

The reader may replace the boundary condition in the definition of $A$ at his own convenience, since from now on everything we do will be completely local.

Let us compute the first variation of the energy $E$ at the point $u$. Using $v:=u+\epsilon \phi$ as test function for the minimality of $u$, where $\epsilon>0$ and $\phi \in H_{0}^{1,2}(\Omega) \cap L^{\infty}(\Omega)$, we obtain that

$$
\begin{gathered}
\int_{\Omega}\left(2 \nabla u \cdot \nabla \phi+\phi \lambda_{+} \chi_{\{u \geqslant-\epsilon \phi\}}-\phi \lambda_{-} \chi_{\{u \leqslant-\epsilon \phi\}}\right) \\
\geqslant-\left|\lambda_{-}\right| \int_{\Omega \cap\{-\epsilon|\phi| \leqslant u \leqslant \epsilon|\phi|\}} \frac{|u|}{\epsilon}-\epsilon \int_{\Omega}|\nabla \phi|^{2}
\end{gathered}
$$

and, as $\epsilon \rightarrow 0$, that

$$
\begin{aligned}
& \int_{\Omega \cap\{u=0\}}\left(-\lambda_{+} \max (\phi, 0)+\lambda_{-} \min (\phi, 0)\right) \\
& \quad \leqslant \int_{\Omega}\left(2 \nabla u \cdot \nabla \phi+\phi \lambda_{+} \chi_{\{u>0\}}-\phi \lambda_{-} \chi_{\{u<0\}}\right) \\
& \leqslant \int_{\Omega \cap\{u=0\}}\left(\lambda_{+} \max (-\phi, 0)-\lambda_{-} \min (-\phi, 0)\right)
\end{aligned}
$$

for every $\phi \in H_{0}^{1,2}(\Omega)$. By the characterization of non-negative distributions this implies that $v \mapsto$ $\int\left(\nabla u \cdot \nabla \phi+\frac{\lambda_{+}}{2} \phi-\frac{\lambda_{-}}{2} \chi_{\{u<0\}} \phi\right)$ is locally in $\Omega$ represented by a finite regular measure. Hence, (2) yields by Radon-Nikodym's theorem that $\Delta u \in L_{\text {loc }}^{1}(\Omega)$ and it follows that $\Delta u=\frac{\lambda_{+}}{2} \chi_{\{u>0\}}-$ $\frac{\lambda_{-}}{2} \chi\{u<0\}$ a.e. in $\Omega$.

Note that for $\lambda_{-} \geqslant 0$ any other function $v \in H^{1,2}(\Omega)$ with boundary data $u_{D}$ on $\partial \Omega$ satisfying the weak equation

$$
\int_{\Omega}\left(2 \nabla v \cdot \nabla \phi+\phi \lambda_{+} \chi_{\{v>0\}}-\phi \lambda_{-} \chi_{\{v<0\}}\right)=0 \quad \text { for every } \phi \in H_{0}^{1,2}(\Omega)
$$


must coincide with $u$. Subtracting the weak equation for $u$ and inserting $\phi:=v-u$ as test function we obtain that

$$
\begin{gathered}
\int_{\Omega} 2|\nabla(v-u)|^{2} \\
\leqslant \int_{\Omega}\left(2 \nabla(v-u) \cdot \nabla(v-u)+\lambda_{+}\left(\chi_{\{v>0\}}-\chi_{\{u>0\}}\right)(v-u)-\lambda_{-}\left(\chi_{\{v<0\}}-\chi_{\{u<0\}}\right)(v-u)\right)=0 .
\end{gathered}
$$

In what follows, the term 'solution' shall always denote a $H^{2,2}$-function solving the strong equation $\Delta v=\frac{\lambda_{+}}{2} \chi_{\{v>0\}}-\frac{\lambda_{-}}{2} \chi_{\{v<0\}}$ a.e. in a given open set.

A powerful tool is now a monotonicity formula introduced in [9] by the author for a class of semilinear free boundary problems (see also [7]). For the sake of completeness let us state the two-phase obstacle problem case here.

TheOREM 3.1 (The MONOTONICITY FORMUla) Suppose that $B_{\delta}\left(x_{0}\right) \subset \Omega$. Then for all $0<$ $\rho<\sigma<\delta$ the function

$$
\begin{gathered}
\Phi_{x_{0}}(r):=r^{-n-2} \int_{B_{r}\left(x_{0}\right)}\left(|\nabla u|^{2}+\lambda_{+} \max (u, 0)+\lambda_{-} \max (-u, 0)\right) \\
-2 r^{-n-3} \int_{\partial B_{r}\left(x_{0}\right)} u^{2} \mathrm{~d} \mathcal{H}^{n-1},
\end{gathered}
$$

defined in $(0, \delta)$ satisfies the monotonicity formula

$$
\Phi_{x_{0}}(\sigma)-\Phi_{x_{0}}(\rho)=\int_{\rho}^{\sigma} r^{-n-2} \int_{\partial B_{r}\left(x_{0}\right)} 2\left(\nabla u \cdot v-2 \frac{u}{r}\right)^{2} \mathrm{~d} \mathcal{H}^{n-1} \mathrm{~d} r \geqslant 0 .
$$

\section{Pointwise regularity and non-degeneracy}

By $L^{p}$-theory the solution $u \in C_{\text {loc }}^{1, \alpha}(\Omega)$ for every $\alpha \in(0,1)$. The set $R:=\Omega \cap\{u=0\} \cap\{\nabla u \neq 0\}$ is therefore open relative to $\Omega \cap(\partial\{u>0\} \cup \partial\{u<0\})$ and the implicit function theorem implies that $R$ is a $C^{1, \alpha}$-surface for every $\alpha \in(0,1)$. The set of interest is therefore the set $S:=\Omega \cap\{\nabla u=$ $0\} \cap(\partial\{u>0\} \cup \partial\{u<0\})$.

LEMMA 4.1 Let $\alpha-1 \in \mathbb{N}$, let $w \in H^{1,2}\left(B_{1}(0)\right)$ be a harmonic function in $B_{1}(0)$ and assume that $D^{j} w(0)=0$ for $0 \leqslant j \leqslant \alpha-1$. Then

$$
\int_{B_{1}(0)}|\nabla w|^{2}-\alpha \int_{\partial B_{1}(0)} w^{2} \mathrm{~d} \mathcal{H}^{n-1} \geqslant 0
$$

and equality implies that $w$ is homogeneous of degree $\alpha$ in $B_{1}(0)$.

Proof. A well known fact first revealed by F. J. Almgren is that the mean frequency

$$
r \mapsto N(r):=r \frac{\int_{B_{r}(0)}|\nabla w|^{2}}{\int_{\partial B_{r}(0)} w^{2} \mathrm{~d} \mathcal{H}^{n-1}}
$$

is non-decreasing in $(0,1)$, and that $N(\sigma)=N(\rho)$ implies for $0<\rho<\sigma<1$ that $w$ is homogeneous of degree $N(\rho)$ in $B_{\sigma}(0)-B_{\rho}(0)$ [4]. Supposing now towards a contradiction that 
$N(s)<\alpha$ for some $s \in(0,1]$, it follows that $\alpha>N(r) \searrow N(0+)$ as $r \searrow 0$. Introducing the scaling $w_{r}(x):=\frac{w(r x)}{\|w(r x)\|_{L^{2}\left(\partial B_{1}(0)\right)}}$, we infer from the boundedness of $N(r)$ and from the compact embedding on the boundary that $w_{r_{m}} \rightarrow w_{0}$ weakly in $H^{1,2}\left(B_{1}(0)\right)$ and $w_{r_{m}} \rightarrow w_{0}$ strongly in $L^{2}\left(\partial B_{1}(0)\right)$ as a certain sequence $r_{m} \rightarrow 0$.

Consequently, the limit $w_{0}$ is a harmonic function in $B_{1}(0)$ satisfying $D^{j} w_{0}(0)=0$ for $0 \leqslant$ $j \leqslant \alpha-1$ and $\left\|w_{0}\right\|_{L^{2}\left(\partial B_{1}(0)\right)}=1$.

Since $N\left(R r_{m}\right) \leqslant N\left(S r_{m}\right) \leqslant N\left(R r_{k}\right)$ for $R \leqslant S$ and $m>>k$, we obtain furthermore that

$$
r \frac{\int_{B_{r}(0)}\left|\nabla w_{0}\right|^{2}}{\int_{\partial B_{r}(0)} w_{0}^{2} \mathrm{~d} \mathcal{H}^{n-1}} \equiv N(0+) \quad \text { for } r \in(0,1),
$$

so $w_{0}$ must be a homogeneous function of degree $N(0+) \in[0, \alpha)$.

The regularity of $w_{0}$ at the point 0 implies that $N(0+) \in \mathbb{N}$. Together with the information that $N(0+)<\alpha$, that $D^{j} w_{0}(0)=0$ for $0 \leqslant j \leqslant \alpha-1$ and that $\left\|w_{0}\right\|_{L^{2}\left(\partial B_{1}(0)\right)}=1$, this yields a contradiction.

Thus $N(s) \geqslant \alpha$ for $s \in(0,1]$, and $N(1)=\alpha$ implies that $N$ is constant on $(0,1)$ and thereby that $w$ is a homogeneous function of degree $\alpha$.

The following proposition gives an estimate on the growth of the solution near $\Omega \cap\{u=0\} \cap$ $\{\nabla u=0\}$

Proposition 4.1 There exists for each $\delta>0$ a constant $C<\infty$ such that

$$
\int_{\partial B_{r}\left(x_{0}\right)} u^{2} \mathrm{~d} \mathcal{H}^{n-1} \leqslant C r^{n-1+4}
$$

for every $r \in(0, \delta)$ and every $x_{0} \in \Omega \cap\{u=0\} \cap\{\nabla u=0\}$ satisfying $B_{2 \delta}\left(x_{0}\right) \in \Omega$.

Furthermore $\Phi_{x_{0}}(r) \geqslant 0$ for every $r \in(0, \delta)$ and every $x_{0} \in \Omega \cap\{u=0\} \cap\{\nabla u=0\}$ satisfying $B_{2 \delta}\left(x_{0}\right) \in \Omega$.

Proof. Let us define the linear space $\Pi:=\left\{p: \mathbb{R}^{n} \rightarrow \mathbb{R}: p\right.$ harmonic and $p$ homogeneous of degree 2\}, let us define for $B_{2 r}\left(x_{0}\right) \subset \Omega$ the function $u_{x_{0}, r}(x):=\frac{u\left(x_{0}+r x\right)}{r^{2}}$ and let us define $p_{x_{0}, r} \in \Pi$ as the orthogonal projection of $u_{x_{0}, r}$ into $\Pi$ with respect to the inner product $(v, w):=$ $\int_{\partial B_{1}(0)} v w \mathrm{~d} \mathcal{H}^{n-1}$.

We maintain that there exists for each $\delta>0$ a constant $C_{1}<\infty$ such that $\operatorname{dist}_{L^{2}\left(\partial B_{1}(0)\right)}\left(u_{x_{0}, r}, \Pi\right) \leqslant C_{1}$ for every $x_{0} \in \Omega \cap\{u=0\} \cap\{\nabla u=0\}$ satisfying $B_{2 \delta}\left(x_{0}\right) \subset \Omega$ and every $r \in(0, \delta)$. Let us assume towards a contradiction that this does not hold. Then there exist sequences $\Omega \cap\{u=0\} \cap\{\nabla u=0\} \ni x_{m} \rightarrow \bar{x}$ and $0<r_{m} \rightarrow 0$ as $m \rightarrow \infty$ such that $\bigcup_{k, m \in \mathbb{N}} B_{2 r_{k}}\left(x_{m}\right) \subset \Omega$ and $M_{m}:=\left\|u_{x_{m}, r_{m}}-p_{x_{m}, r_{m}}\right\|_{L^{2}\left(\partial B_{1}(0)\right)} \rightarrow+\infty$ as $m \rightarrow \infty$. Introducing $u_{m}:=u_{x_{m}, r_{m}}, p_{m}:=p_{x_{m}, r_{m}}$ and $v_{m}:=\frac{u_{m}-p_{m}}{M_{m}}$ we obtain from the monotonicity formula Theorem 3.1 (using in the case $\lambda_{-}<0$ the fact that $u_{m}$ is subharmonic in $B_{1}(0)$ and $u_{m}(0)=0$ and the fact that therefore $\int_{B_{1}(0)}\left(\lambda_{+} \max \left(u_{m}, 0\right)+\lambda_{-} \max \left(-u_{m}, 0\right)\right)=\lambda_{+} \int_{B_{1}(0)} u_{m}+$ 
$\left.\left(\lambda_{+}+\lambda_{-}\right) \int_{B_{1}(0)} \max \left(-u_{m}, 0\right) \geqslant 0\right)$ that

$$
\begin{gathered}
\int_{B_{1}(0)}\left|\nabla v_{m}\right|^{2}-2 \int_{\partial B_{1}(0)} v_{m}^{2} \mathrm{~d} \mathcal{H}^{n-1} \leqslant \frac{1}{M_{m}^{2}} \Phi_{x_{m}}\left(r_{m}\right) \\
+\frac{1}{M_{m}^{2}} \int_{\partial B_{1}(0)}\left(p_{m} \nabla p_{m} \cdot v-2 u_{m} \nabla p_{m} \cdot v-2 p_{m}{ }^{2}+4 u_{m} p_{m}\right) \mathrm{d} \mathcal{H}^{n-1} \\
\leqslant \frac{1}{M_{m}^{2}} \Phi_{x_{m}}\left(\sup _{k \in \mathbb{N}} r_{k}\right) \rightarrow 0
\end{gathered}
$$

as $m \rightarrow \infty$. Passing to a subsequence $m \rightarrow \infty$ such that $v_{m} \rightarrow v$ weakly in $H^{1,2}\left(B_{1}(0)\right)$ as $m \rightarrow \infty$, the compact embedding on the boundary implies that $\|v\|_{L^{2}\left(\partial B_{1}(0)\right)}=1$, that

$$
\int_{B_{1}(0)}|\nabla v|^{2} \leqslant 2 \int_{\partial B_{1}(0)} v^{2} \mathrm{~d} \mathcal{H}^{n-1}
$$

and that

$$
\int_{\partial B_{1}(0)} v p \mathrm{~d} \mathcal{H}^{n-1}=0 \quad \text { for every } p \in \Pi \text {. }
$$

Since

$$
\Delta v_{m}=\frac{1}{M_{m}}\left(\frac{\lambda_{+}}{2} \chi_{\left\{u_{m}>0\right\}}-\frac{\lambda_{-}}{2} \chi_{\left\{u_{m}<0\right\}}\right),
$$

it follows that $v$ is harmonic in $B_{1}(0)$. Moreover, we obtain from $L^{p}$-theory that $v_{m} \rightarrow v$ in $C_{\mathrm{loc}}^{1, \alpha}\left(B_{1}(0)\right)$ for each $\alpha \in(0,1)$ as $m \rightarrow \infty$. Consequently $v(0)=0$ and $\nabla v(0)=0$.

Thus we can apply Lemma 4.1 with respect to $\alpha=2$ and obtain from (3) that $v$ is homogeneous of degree 2, contradicting (4) as $\|v\|_{L^{2}\left(\partial B_{1}(0)\right)}=1$.

We infer from this contradiction that

$$
\begin{gathered}
\int_{B_{1}(0)}\left(\lambda_{+} \max \left(u_{x_{0}, r}, 0\right)+\lambda_{-} \max \left(-u_{x_{0}, r}, 0\right)\right)=\Phi_{x_{0}}(r)-\int_{B_{1}(0)}\left|\nabla\left(u_{x_{0}, r}-p_{x_{0}, r}\right)\right|^{2} \\
+2 \int_{\partial B_{1}(0)}\left(u_{x_{0}, r}-p_{x_{0}, r}\right)^{2} \mathrm{~d} \mathcal{H}^{n-1} \leqslant \Phi_{x_{0}}(\delta)+2 C_{1}{ }^{2}
\end{gathered}
$$

for every $r \in(0, \delta)$ and every $x_{0} \in \Omega \cap\{u=0\} \cap\{\nabla u=0\}$ satisfying $B_{2 \delta}\left(x_{0}\right) \subset \Omega$. In the case that $\lambda_{-}<0$ we observe that $u_{x_{0}, r}$ is subharmonic in $B_{1}(0)$ and vanishes at the origin and satisfies therefore $\int_{B_{1}(0)} u_{x_{0}, r} \geqslant 0$. Consequently the left-hand side

$$
\begin{gathered}
\int_{B_{1}(0)}\left(\lambda_{+} \max \left(u_{x_{0}, r}, 0\right)+\lambda_{-} \max \left(-u_{x_{0}, r}, 0\right)\right) \\
\geqslant\left(\lambda_{+}+\lambda_{-}\right) \max \left(\int_{B_{1}(0)} \max \left(u_{x_{0}, r}, 0\right), \int_{B_{1}(0)} \max \left(-u_{x_{0}, r}, 0\right)\right) \\
\geqslant \frac{\lambda_{+}+\lambda_{-}}{2} \int_{B_{1}(0)}\left|u_{x_{0}, r}\right|
\end{gathered}
$$


for $x_{0}$ and $r$ as above, which finishes the proof of our first statement.

Suppose now that $\lambda_{-} \geqslant 0$ and that $0<r_{m} \rightarrow 0$ and $\Omega \cap\{u=0\} \cap\{\nabla u=0\} \ni x_{m} \rightarrow \bar{x}$ as $m \rightarrow \infty$, that $\bigcup_{k, m \in \mathbb{N}} B_{2 r_{k}}\left(x_{m}\right) \subset \Omega$ and that $N_{M}:=\left\|u_{x_{m}, r_{m}}\right\|_{L^{2}\left(\partial B_{1}(0)\right)} \rightarrow+\infty$ as $m \rightarrow \infty$. As before, we obtain that some limit $w$ of $\frac{u_{x_{m}, r_{m}}}{N_{m}}$ is harmonic in $B_{1}(0)$ and satisfies $w(0)=0$ and $\|w\|_{L^{2}\left(\partial B_{1}(0)\right)}=1$. On the other hand we infer from the above estimate for $\int_{B_{1}(0)}\left(\lambda_{+} \max \left(u_{x_{m}, r_{m}}, 0\right)+\lambda_{-} \max \left(-u_{x_{m}, r_{m}}, 0\right)\right)$ that $w \leqslant 0$ in the case of $\lambda_{+}>0$ and that $w \geqslant 0$ in the case of $\lambda_{-}>0$ which leads by the strong maximum principle to a contradiction. Thus the first statement of our proposition has been proved.

Furthermore, this puts us in a position to show that $\Phi_{x_{0}}(r) \geqslant 0$ for every $r>0$ and $x_{0} \in$ $\Omega \cap\{u=0\} \cap\{\nabla u=0\}$ satisfying $B_{r}\left(x_{0}\right) \subset \Omega$.

The function $r \mapsto \Phi_{x_{0}}(r)$, being bounded and non-decreasing, has a right limit $\Phi_{x_{0}}(0+)$ as $r \searrow 0$, and we obtain from the monotonicity formula Theorem 3.1 that $0 \leftarrow \Phi_{x_{0}}(\sigma)-\Phi_{x_{0}}(\rho)=$ $\int_{\rho}^{\sigma} s^{-n-2} \int_{\partial B_{s}(0)} 2\left(\nabla u \cdot v-2 \frac{u}{s}\right)^{2} \mathrm{~d} \mathcal{H}^{n-1} \mathrm{~d} s$ as $0<\rho<\sigma \rightarrow 0$. Supposing now that $\Phi_{x_{0}}(r) \searrow$ $\Phi_{x_{0}}(0+)<0$ as $r \searrow 0$, we obtain a sequence $r_{m} \rightarrow 0$ such that $u_{x_{0}, r_{m}} \rightarrow u_{0}$ in $C_{\text {loc }}^{1, \alpha}\left(\mathbb{R}^{n}\right)$ and $u_{0}$ is a homogeneous solution of degree 2 . This leads to the contradiction

$$
\begin{gathered}
0>\Phi_{x_{0}}(0+)=\int_{B_{1}(0)}\left(\left|\nabla u_{0}\right|^{2}+\lambda_{+} \max \left(u_{0}, 0\right)+\lambda_{-} \max \left(-u_{0}, 0\right)\right) \\
-2 \int_{\partial B_{1}(0)} u_{0}^{2} \mathrm{~d} \mathcal{H}^{n-1}=\int_{B_{1}(0)}\left(\frac{\lambda_{+}}{2} \max \left(u_{0}, 0\right)-\frac{\lambda_{-}}{2} \min \left(u_{0}, 0\right)\right) \geqslant 0 .
\end{gathered}
$$

LEMMA 4.2 (NON-DEGENERACY) For every $x_{0} \in \Omega \cap(\partial\{u>0\} \cup \partial\{u<0\})$ and every $B_{2 r}\left(x_{0}\right) \subset$ $\Omega$ the estimate

$$
\sup _{\partial B_{r}\left(x_{0}\right)}|u| \geqslant \frac{1}{4 n} \min \left(\lambda_{+}, \lambda_{-}\right) r^{2} \quad \text { holds for } \min \left(\lambda_{-}, \lambda_{+}\right)>0
$$

and the estimate

$$
\sup _{\partial B_{r}\left(x_{0}\right)}|u| \geqslant \frac{1}{4 n} \max \left(\lambda_{+}, \lambda_{-}\right) r^{2} \quad \text { holds for } \min \left(\lambda_{-}, \lambda_{+}\right) \leqslant 0 .
$$

Proof. Let us first assume that $\lambda_{+}>0$.

Considering now first a point $x_{0} \in \partial\{u>0\}$ such that $B_{2 r}\left(x_{0}\right) \subset \Omega$, we choose a sequence $\{u>0\} \ni x_{m} \rightarrow x_{0}$ as $m \rightarrow \infty$. Supposing that $\sup _{\partial B_{r}\left(x_{m}\right)} u \leqslant \frac{1}{4 n} \lambda_{+} r^{2}$, the comparison principle yields that $u(x) \leqslant v(x):=\frac{1}{4 n} \lambda_{+}\left|x-x_{m}\right|^{2}$ in $B_{r}\left(x_{m}\right)$, a contradiction to the fact that $u\left(x_{m}\right)>0$.

Next we consider a point $x_{0} \in \partial\{u<0\} \cap\{\max (u, 0)=0\}^{\circ}$ such that $B_{2 r}\left(x_{0}\right) \subset \Omega$. In the case that $\lambda_{-} \leqslant 0$ this yields immediately a contradiction to the strong maximum principle, as $\min (u, 0)$ is subharmonic in a neighbourhood of the point $x_{0}$. In the case that $\lambda_{-}>0, x_{0} \in \partial\{u<0\}$ and $B_{2 r}\left(x_{0}\right) \subset \Omega$ we repeat the above comparison argument to obtain

$$
\sup _{\partial B_{r}\left(x_{0}\right)}-u \geqslant \frac{1}{4 n} \lambda_{-} r^{2}
$$

This finishes the proof for $\lambda_{+}>0$.

In the case that $\lambda_{+}=0$, it follows that $\lambda_{-}>0$, and we apply the already proven estimate to the solution $-u$. 
REMARK 4.1 Thus $\Omega \cap(\partial\{u>0\} \cup \partial\{u<0\})$ can be decomposed into four parts: the regular part $P_{1}$ where $\nabla u \neq 0$, the one-phase parts $P_{2}:=\Omega \cap \partial\{u>0\}-\partial\{u<0\}$ and $P_{3}:=\Omega \cap \partial\{u<$ $0\}-\partial\{u>0\}$ to which the existing regularity theory can be applied (see, for example, [1] and [7]) and $P_{4}:=\Omega \cap \partial\{u<0\} \cap \partial\{u>0\} \cap\{\nabla u=0\}$ at which $\nabla u$ satisfies a linear growth condition. Concerning $P_{4}$ one obtains from a combination of our growth estimate with the well known estimate

$$
\sup _{B_{\frac{1}{2}}(0)}|\nabla v|^{2} \leqslant C(n) \sup _{B_{1}(0)} v^{2}
$$

(which holds for every harmonic function defined in $B_{1}(0)$ ) by a scaling and covering argument that $u \in C^{1,1}(U)$ for some open neighbourhood $U$ of the interior of $P_{4}$ relative to $\Omega \cap(\partial\{u>0\} \cup \partial\{u<$ $0\})$. It should therefore be possible to obtain regularity of the relative interior of $P_{4}$, which will not be investigated in this paper. Here we will derive a Hausdorff dimension estimate for the entire free boundary $\Omega \cap(\partial\{u>0\} \cup \partial\{u<0\})$.

\section{A Hausdorff dimension estimate}

LEMmA 5.1 Let $x_{0} \in S$ and let $u_{k}(x):=\frac{u\left(x_{0}+\rho_{k} x\right)}{\rho_{k}{ }^{2}}$ be a blow-up sequence: i.e. assume that $\rho_{k} \rightarrow 0$ as $k \rightarrow \infty$. Then $\left(u_{k}\right)_{k \in \mathbb{N}}$ is for each open $D \subset \subset \mathbb{R}^{n}$ and each $p \in(1, \infty)$ bounded in $H^{2, p}(D)$, and each limit $u_{0}$ with respect to a subsequence $k \rightarrow \infty$ is a nontrivial homogeneous solution of degree 2 in $\mathbb{R}^{n}$ and satisfies the following condition: for each compact set $K \subset \mathbb{R}^{n}$ and each open set $U \supset K \cap S_{0}$ there exists $k_{0}<\infty$ such that $S_{k} \cap K \subset U$ for $k \geqslant k_{0}$; here $S_{0}:=\left\{\nabla u_{0}=\right.$ $0\} \cap\left(\partial\left\{u_{0}>0\right\} \cup \partial\left\{u_{0}<0\right\}\right)$ and $S_{k}:=\left\{\nabla u_{k}=0\right\} \cap\left(\partial\left\{u_{k}>0\right\} \cup \partial\left\{u_{k}<0\right\}\right)$.

Proof. The boundedness of the sequence follows from Proposition 4.1, the nontriviality of the limit follows from Lemma 4.2 and the homogeneity of the limit follows from the monotonicity formula Theorem 3.1 and from the fact that $\Phi_{x_{0}}\left(\rho_{k}\right) \rightarrow \Phi_{x_{0}}(0+)$ as $k \rightarrow \infty$. Concerning the last statement let us suppose that $S_{k} \cap(K-U) \ni x_{k} \rightarrow \bar{x}$ as $k \rightarrow \infty$. Then $\bar{x} \in\left\{u_{0}=0\right\} \cap\left\{\nabla u_{0}=0\right\} \cap(K-U)$, and the assumption $U \supset K \cap S_{0}$ implies that $u_{0}=0$ on $\overline{B_{\delta}(\bar{x})}$ for some $\delta>0$. The uniform convergence of $u_{k}$ to $u_{0}$ on $\overline{B_{\delta}(\bar{x})}$ as well as the non-degeneracy of $u_{k}$ (Lemma 4.2) lead therefore to a contradiction for large $k$.

THEOREM 5.1 The Hausdorff dimension of the set $S$ is less than or equal to $n-1$.

Proof. Suppose that $m>n-1$ and that $\mathcal{H}^{m}(S)>0$. Then we may use [5: Proposition 11.3], Lemma 5.1 as well as [5: Lemma 11.5] at $\mathcal{H}^{m}$-a.e. point of $S$ to obtain a blow-up limit $u_{0}$ with the properties mentioned in Lemma 5.1, satisfying $\mathcal{H}^{m, \infty}\left(S_{0}\right)>0$. By [5: Lemma 11.2] we find a point $\bar{x} \in S_{0}-\{0\}$ at which the density in [5: Proposition 11.3] is estimated from below. Now each blow-up limit $u_{00}$ with respect to $\bar{x}$ (and with respect to a subsequence $k \rightarrow \infty$ such that the limit superior in [5: Proposition 11.3] becomes a limit) again satisfies the properties of Lemma 5.1; in addition, we obtain from the homogeneity of $u_{0}$ as in Lemma 3.1 of [8] that the rotated $u_{00}$ is constant in the direction of the $n$th unit vector. Defining $\bar{u}$ as the restriction of this rotated $u_{00}$ to $\mathbb{R}^{n-1}$, it follows therefore that $\mathcal{H}^{m-1}((\partial\{\bar{u}>0\} \cup \partial\{\bar{u}<0\}) \cap\{\nabla \bar{u}=0\})>0$.

Repeating this procedure $n-2$ times we obtain a nontrivial homogeneous solution $u^{\star}$ of degree 2 in $\mathbb{R}$ satisfying $\mathcal{H}^{m-(n-1)}\left(\left(\partial\left\{u^{\star}>0\right\} \cup \partial\left\{u^{\star}<0\right\}\right) \cap\left\{\nabla u^{\star}=0\right\}\right)>0$, a contradiction.

COROLLARY 5.1 The Hausdorff dimension of $\partial\{u>0\} \cup \partial\{u<0\}$ is less than or equal to $n-1$.

REMARK 5.1 Proposition 4.1 and Corollary 5.1 can by obvious modifications be extended to the case of Hölder continuous coefficients $\lambda_{+}$and $\lambda_{-}$satisfying the assumptions in Section 3. 


\section{REFERENCES}

1. Caffarelli, L. A. The regularity of free boundaries in higher dimensions. Acta Math. 139, (1977) $155-184$.

2. CAnNon, J. R. \& Hill, C. D. On the movement of a chemical reaction interface. Indiana Univ. Math. J. 20, (1970) 429-454.

3. Evans, L. A chemical diffusion-reaction free boundary problem. Nonlinear Anal. 6, (1982) 455-466.

4. Garofalo, N. \& Lin, F. H. Monotonicity properties of variational integrals, $A_{p}$ weights and unique continuation. Indiana Univ. Math. J. 35, (1986) 245-268.

5. Giusti, E. Minimal Surfaces and Functions of Bounded Variation. Birkhäuser, Boston (1984).

6. Tonegawa, Y. On the regularity of a chemical reaction interface. Commun. Partial Diff. Eqns 23, (1998) 1181-1207.

7. WeISs, G. S. A homogeneity improvement approach to the obstacle problem. Inv. Math. 138, (1999) 23-50.

8. WeISS, G. S. Partial regularity for a minimum problem with free boundary. J. Geom. Anal. 9, (1999) 317-326.

9. WeIss, G. S. Partial regularity for weak solutions of an elliptic free boundary problem. Commun. Partial Diff. Eqns 23, (1998) 439-455. 